\title{
Guided chemotherapy based on patient-derived mini-xenograft models improves survival of gallbladder carcinoma patients
}

Ming Zhan ${ }^{1 \dagger}$, Rui-meng Yang ${ }^{1 \dagger}$, Hui Wang ${ }^{1}$, Min He${ }^{1}$, Wei Chen ${ }^{1}$, Sun-wang Xu' ${ }^{1}$ Lin-hua Yang ${ }^{1}$, Qiang Liư ${ }^{2}$, Man-mei Long ${ }^{3}$ and Jian Wang ${ }^{1 *}$

\begin{abstract}
Background: Gallbladder carcinoma is highly aggressive and resistant to chemotherapy, with no consistent strategy to guide first line chemotherapy. However, patient-derived xenograft (PDX) model has been increasingly used as an effective model for in preclinical study of chemosensitivity.

Methods: Mini-PDX model was established using freshly resected primary lesions from 12 patients with gallbladder to examine the sensitivity with five of the most commonly used chemotherapeutic agents, namely gemcitabine, oxaliplatin, 5-fluorouracil, nanoparticle albumin-bound (nab)-paclitaxel, and irinotecan. The results were used to guide the selection of chemotherapeutic agents for adjunctive treatment after the surgery. Kaplan-Meier method was used to compare overall survival (OS) and disease free survival (DFS) with 45 patients who received conventional chemotherapy with gemcitabine and oxaliplatin.

Results: Cell viability assays based on mini-PDX model revealed significant heterogeneities in drug responsiveness. Kaplan-Meier analysis showed that patients in the PDX-guided chemotherapy group had significantly longer median OS (18.6 months; $95 \% \mathrm{Cl} 15.9-21.3$ months) than patients in the conventional chemotherapy group (13.9 months; 95\% Cl 11.7-16.2 months) ( $P=0.030 ; \mathrm{HR} 3.18 ; 95 \% \mathrm{Cl} 1.47-6.91)$. Patients in the PDX-guided chemotherapy group also had significantly longer median DFS (17.6 months; $95 \% \mathrm{Cl} 14.5-20.6$ months) than patients in the conventional chemotherapy group (12.0 months; 95\% Cl 9.7-14.4 months) ( $P=0.014 ; \mathrm{HR} 3.37$; 95\% Cl 1.67-6.79).
\end{abstract}

Conclusion: The use of mini-PDX model to guide selection of chemotherapeutic regimens could improve the outcome in patients with gallbladder carcinoma.

Keywords: Gallbladder cancer, Mini-PDX, Chemosensitivity, Overall survival, Personalized therapy

\footnotetext{
*Correspondence: dr_wangjianrenji@163.com

${ }^{\dagger}$ Ming Zhan and Rui-meng Yang contributed equally to this work

${ }^{1}$ Department of Biliary-Pancreatic Surgery, Renji Hospital, School

of Medicine, Shanghai Jiao Tong University, 160 Pujian Road,

Shanghai 200127, P. R. China

Full list of author information is available at the end of the article
}

(c) The Author(s) 2018. This article is distributed under the terms of the Creative Commons Attribution 4.0 International License (http://creativecommons.org/licenses/by/4.0/), which permits unrestricted use, distribution, and reproduction in any medium, provided you give appropriate credit to the original author(s) and the source, provide a link to the Creative Commons license, and indicate if changes were made. The Creative Commons Public Domain Dedication waiver (http://creativecommons.org/ publicdomain/zero/1.0/) applies to the data made available in this article, unless otherwise stated. 


\section{Introduction}

Gallbladder carcinoma, the most common biliary tract malignancy, is characterized by its aggressive growth and high lethality [1]. The disease generally carries a dismal prognosis due to its advanced stage at initial diagnosis and its recalcitrance to treatment $[2,3]$. Despite advances in therapeutic strategies against gallbladder neoplastic disorders, surgical resection in combination with neoadjuvant or adjuvant therapies still remains the optimal treatment modality [4]. Unfortunately, only a small proportion of gallbladder carcinoma patients are eligible for surgical intervention. Despite the controversial role of adjuvant therapy, a multimodal therapeutic approach may benefit patients at high risk for recurrence, such as for those with lymph node metastasis or positive resection margins [5].

Recently, several new cytotoxic agents have been proven effective for advanced biliary tract cancer, with a reduction in the rate of morbidity and mortality [6]. A number of clinical trials are underway to examine the effectivity of adjuvant chemotherapy with gemcitabine, capecitabine, or S-1 in combination with platinum. Two phase III trials have shown that the combination of gemcitabine with cisplatin or oxaliplatin is superior to singleagent chemotherapy in improving the overall survival (OS) of biliary tract cancer patients and is now used as the standard palliative regimen [7]. Several phase II studies have also investigated the efficacy of targeted agents against EGFR, VEGF, HER2, and MEK [8].

Irinotecan is a camptothecin derivative that exerts antitumor activities against a variety of tumor types by targeting topoisomerase I, consequently leading to the formation of DNA double-strand breaks and inhibition of DNA synthesis. A retrospective study in patients with advanced biliary tract cancer suggested that the combination therapy of irinotecan with gemcitabine and fluorouracil confers promising survival benefits with manageable toxicities [9]. In addition, a phase II trial of gemcitabine in combination with irinotecan indicated comparable efficacy with historic control [10]. However, given the rarity of these tumors, evidence is still largely based on retrospective studies, surveillance, epidemiological, and end results database inquiries, single or multi-institutional prospective studies, and meta-analysis of studies on adjuvant therapy [11]. Therefore, systematic prospective investigations are urgently needed.

Continued efforts in gene expression profiling and genomic sequencing have uncovered the underlying complexity and molecular heterogeneity of gallbladder carcinoma, shedding light on the daunting challenges of therapeutic interventions [12]. The highly intrinsic heterogeneity of gallbladder carcinoma and varied responses to chemotherapeutic drugs mandate a personalized approach to gallbladder carcinoma treatment [13]. Recently, there have been increasing interests in the development and characterization of patient-derived xenograft (PDX) tumor models for cancer research [14]. PDX models retain the principal histologic and genetic characteristics of donor tumors and remain stable throughout passages. These models have been shown to be predictive of clinical outcomes and are used for preclinical drug evaluation, biomarker identification, biological studies, and personalized medicine strategies [15-17]. Thus, PDX models are useful in recapitulating the complexity and heterogeneity of gallbladder carcinoma. However, there are limitations for direct application of traditional PDX models on gallbladder carcinoma patients. It typically takes $4-8$ months for an established PDX model to be ready for assessing drug sensitivity, which is unduly long for initiation of drugsensitivity guided treatment of gallbladder carcinoma patients. Mini-PDX model offers an effective alternative as it only takes about 7 days for the model to complete drug sensitivity test and could thus provide guidance for prompt personalized selection of individual drugs for each patient.

In the present study, we first established a mini-PDX model using fresh primary tumor cells from 12 surgically resected gallbladder carcinomas. Responses to five of the most commonly used chemotherapeutic agents including gemcitabine, oxaliplatin, 5-fluorouracil, nanoparticle albumin-bound (nab)-paclitaxel, and irinotecan were examined. We treated 12 gallbladder carcinoma patients with the top two efficient agents as obtained from the results of drug sensitivity tests. Patient outcomes then were compared with 45 gallbladder carcinoma patients who were conventionally treated with gemcitabine and oxaliplatin. Our results demonstrated that drug sensitivity-guided chemotherapy yielded significantly better outcomes as revealed by increased OS and disease free survival rate (DFS) than conventional chemotherapy. Analysis of the clinicopathologic features of gallbladder carcinoma patients further revealed that gemcitabine sensitivity was associated with nerve invasion while irinotecan sensitivity was associated with tumor size, lymph node metastasis and TNM stage. Lastly, we also found strong association between responses to drug sensitivityguided therapy and the expression of several important chemoresistance-related proteins (e.g., p53 and $P$-gp).

\section{Materials and methods}

\section{Tissue specimen acquisition}

Fresh tissue specimens were obtained from 57 treatment naïve gallbladder carcinoma patients who underwent surgery at the Department of Biliary-Pancreatic Surgery of Renji Hospital, School of Medicine, Shanghai Jiao 
Tong University between September 2014 and September 2016. Gallbladder carcinoma was staged according to the TNM classification (AJCC 7th edition).

\section{Follow-up}

Follow-ups were conducted once every month during the first year post surgery and once every 3 months thereafter. Phone calls were made to patients and their relatives according to the follow-up guidelines of the National Comprehensive Cancer Network of China. OS was calculated from the date of surgery until the date of the final follow-up visit or death and DFS was calculated from the date of surgery until the final follow-up visit or tumor recurrence. The final follow-up visit was September 2017.

\section{Mini-PDX models and drug sensitivity assays}

Mini-PDX models were established using freshly removed gallbladder carcinoma tissues from 12 patients. Drug sensitivity was examined using the OncoVee ${ }^{\mathrm{TM}}$-Mini PDX assay (LIDE Biotech, Shanghai, China). Briefly, gallbladder carcinoma samples were washed with Hank's balanced salt solution (HBSS) to remove non-tumor tissues and necrotic tumor tissues. After morselization, the tumor tissues were digested with collagenase at $37^{\circ} \mathrm{C}$ for 1-2 h. Cells were collected followed by removal of blood cells and fibroblasts. Then, gallbladder carcinoma cell suspension was transferred to the HBSS washed capsules.

Four-weeks-old BALB/c nude mice (SLARC Inc., Shanghai, China), weighing 15-20 g each, were used for subcutaneous implantation. A small skin incision was made and the capsule was embedded in the subcutaneous tissue. Generally, each mouse received 3 capsules. Drugs (gemcitabine, $60 \mathrm{mg} / \mathrm{kg}$, IP, Q4D $\times 2$; oxaliplatin, $5 \mathrm{mg} /$ $\mathrm{kg}$, IP, Q4D $\times 2$; 5 -fluorouracil, $25 \mathrm{mg} / \mathrm{kg}, \mathrm{IP}, \mathrm{QD} \times 5$; nab-paclitaxel, $20 \mathrm{mg} / \mathrm{kg}$, IV, QD $\times 5$; irinotecan, $50 \mathrm{mg} /$ $\mathrm{kg}, \mathrm{IP}, \mathrm{Q} 4 \mathrm{D} \times 2$ ) were administered for 7 days respectively. Normal saline was used as the control. Anti-tumor activity was evaluated based on the relative fluorescence units (RFU) using the CellTiter-Glo ${ }^{\circledR}$ Luminescent Cell Viability Assay (Promega, Madison, WI, USA). Proliferation rate was calculated using the equation:

Proliferation rate $=\left(\mathrm{RFU}^{\mathrm{D} 7}-\mathrm{RFU}^{\mathrm{D} 0}\right)_{\mathrm{drug}} /\left(\mathrm{RFU}^{\mathrm{D} 7}-\mathrm{RFU}^{\mathrm{D} 0}\right)_{\text {placebo }}$

The study flowchart is shown in Fig. 1a. All procedures were performed under specific pathogen free conditions and carried out in accordance with the guidelines for the Care and Use of Laboratory Animals of the National Institutes of Health.

\section{Immunohistochemistry}

Formalin-fixed, paraffin-embedded tissues were immunohistochemically stained as described previously [18]. Primary antibodies against the following proteins were used: p53 (1:200, ab1101, Abcam, Cambridge, UK), Ki-67 (1:600, ab15580, Abcam), P-gp (1:800, 13978, CST, MA, USA), MRP1 (1:200, 72202, CST), Bcl2 (1:400, 15071, CST), TS (1:100, ab58287, Abcam), GST- $\pi$ (1:200, ab58287, Abcam), and Bcl-2 (1:400, 12286, CST). Immunohistochemical staining was semi-quantitatively scored by rating staining intensity of a protein of interest (I: negative, 0 ; weak, 1 ; moderate, 2 ; intense, 3 ) and the percentage of positively stained cells (P: $0 \%-5 \%$, scored 0; $6 \%-35 \%$, scored $1 ; 36 \%-70 \%$, scored 2 ; and $>70 \%$, scored 3) to obtain a final score $(Q)$, which was defined as the product of $\mathrm{I} \times \mathrm{P}$. Two senior pathologists evaluated the tissues independently in a blinded manner.

\section{Statistical analysis}

Data are presented as mean \pm standard deviation (SD). Normally distributed continuous variables were analyzed using unpaired Student's $t$-test. For multiple comparisons, the Tukey-Kramer honestly significant difference test was applied following ANOVA. KaplanMeier method and log-rank test were used to analyze OS and DFS. Data were censored for patients who were lost to follow-up. Pearson $\chi^{2}$ test was used to analyze the correlation between clinicopathological variables and drug sensitivity. SPSS 17.0 software (SPSS Inc., Chicago, IL, USA) was used for all statistical analyses. For all analysis, $P<0.05$ was considered statistically significant.

\section{Ethical approval}

The present study was approved by the Ethical Committee of Renji Hospital, School of Medicine, Shanghai Jiao Tong University. Written informed consents were provided by all participants before enrollment. All procedures were performed in accordance with the Ethical Standards of Institutional/National Research Committees and the 1964 Helsinki Declaration, its later amendments, or similar ethical standards.

\section{Results}

The mini-PDX model yields drug sensitivity patterns of gallbladder carcinoma patients

Cell viability assays showed that the mean proliferation rate of gallbladder carcinoma cells treated with gemcitabine, oxaliplatin, 5-fluorouracil, nab-paclitaxel, or irinotecan was $46.1 \%, 69.8 \%, 69.7 \%, 59.9 \%$, and $43.0 \%$, respectively (Fig. 1b). Drug sensitivity varied substantially, with the highest relative proliferation rate at 


\section{a}

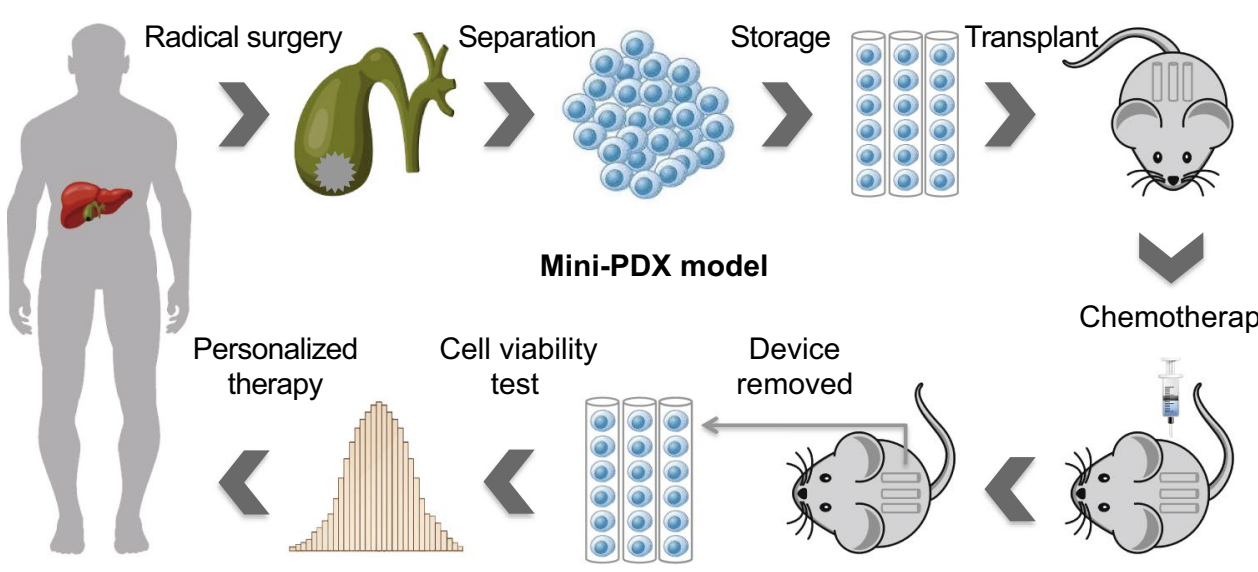

b

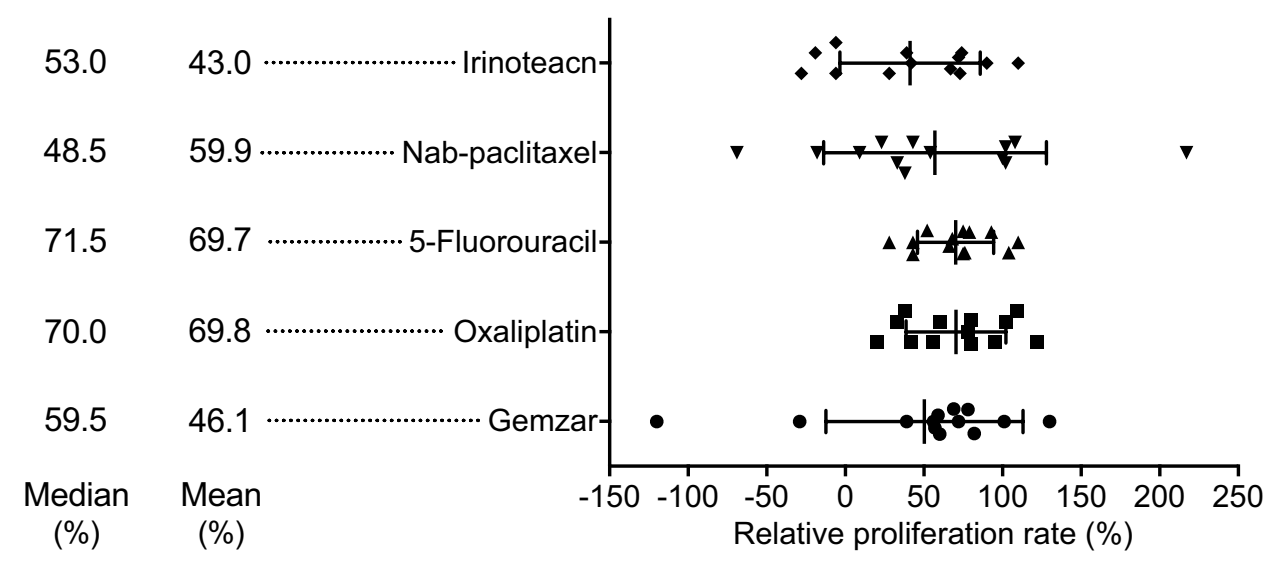

C

\begin{tabular}{|c|c|c|c|c|c|c|c|c|c|c|c|c|}
\hline Groups & & & & & Chen & nothera & apy prc & grams & & & & \\
\hline \multirow{2}{*}{ Mini-PDX } & NO.1 & NO.2 & NO.3 & NO.4 & NO.5 & NO.6 & NO.7 & NO.8 & NO.9 & NO.10 & NO.11 & NO.12 \\
\hline & $1+3$ & $3+5$ & $1+2$ & $2+5$ & $1+5$ & $2+3$ & $3+5$ & $2+4$ & $2+5$ & $1+5$ & $2+4$ & $1+2$ \\
\hline Conventiona & \multicolumn{12}{|c|}{$4+5$} \\
\hline
\end{tabular}

1: Irinoteacn; 2: Nab-paclitaxel; 3: 5-Fluorouracil; 4: Oxaliplatin; 5: Gemzar

Fig. 1 An overview of the generation of the mini-PDX model. a Gallbladder carcinoma cells from gallbladder carcinoma patients under surgical resection were transferred to the HBSS washed capsules and then subcutaneously implanted in BALB/c nude mice. Drugs or placebo (saline) were injected via the tail veins or intraperitoneally. Finally, the capsules were taken out and the anti-tumor activity was evaluated by detecting cell viabilities via CTG assays. Based on the anti-tumor activity data of the mini-PDX models, the optimal chemotherapy regimens were selected for different gallbladder carcinoma patients. $\mathbf{b}$ Scatter plot shows the results of the relative proliferation rate of the five drugs tested on the mini-PDX model among the 12 gallbladder carcinoma patients. c Detailed results reveal the two most effective agents chosen for treating the patients in the mini-PDX group and the conventional chemotherapy group

$110 \%$ and the lowest at $28 \%$ of irinotecan implying the requirement of individualized therapy (Fig. 1b). The agents used in the investigated patients are shown in Fig. 1c.
Mini-PDX-guided chemotherapy is superior to conventional chemotherapy in prolonging survival of gallbladder carcinoma patients

The cohort included 24 males and 33 females with a mean age $66.6 \pm 9.5$ years. Patients in the PDX-guided 
chemotherapy group included 5 male (41.7\%) and 7 female patients $(58.3 \%)$ with a median age of 67 years (range 56-87 years). The PDX-guided chemotherapy group and the conventional chemotherapy group had comparable demographic and baseline characteristics (Table 1). Kaplan-Meier analysis showed that patients in the PDX-guided chemotherapy group had significantly longer median OS (18.6 months; $95 \% \mathrm{CI}$ 15.9-21.3 months) than patients in the conventional chemotherapy group (13.9 months; 95\% CI 11.716.2 months) $(P=0.030$; HR 3.18 ; 95\% CI $1.47-6.91)$ (Fig. 2a). Patients in PDX-guided chemotherapy group

Table 1 Patient demographic and baseline characteristics

\begin{tabular}{|c|c|c|c|c|c|}
\hline Characteristic & All & $\begin{array}{l}\text { Conventional } \\
\text { chemotherapy }\end{array}$ & $\begin{array}{l}\text { PDX-guided } \\
\text { chemotherapy }\end{array}$ & OR $(95 \% \mathrm{Cl})$ & $P^{\mathbf{a}}$ \\
\hline No. & 57 & 45 & 12 & & \\
\hline Female gender, n (\%) & $33(57.89)$ & $26(57.78)$ & $7(58.33)$ & $1.02(0.28-3.72)$ & 0.972 \\
\hline \multicolumn{6}{|l|}{ Age, years, n (\%) } \\
\hline$<65$ & $23(40.35)$ & $18(40)$ & $5(41.67)$ & $1.07(0.29-3.91)$ & 0.917 \\
\hline \multicolumn{6}{|l|}{ Gallstone, n (\%) } \\
\hline Yes & 44 (77.19) & $36(80)$ & $8(66.67)$ & $0.5(0.12-2.04)$ & 0.555 \\
\hline \multicolumn{6}{|l|}{ CA19-9, U/mL, n (\%) } \\
\hline$<37$ & $16(28.07)$ & $13(28.89)$ & $3(25)$ & $0.82(0.19-3.52)$ & 0.924 \\
\hline \multicolumn{6}{|l|}{ Tumor size, cm, n (\%) } \\
\hline$<4$ & $37(64.91)$ & $31(68.89)$ & $6(50)$ & $0.45(0.12-1.65)$ & 0.223 \\
\hline \multicolumn{6}{|c|}{ Tumor differentiation, n (\%) } \\
\hline Well and moderate & $31(54.39)$ & $23(51.11)$ & $8(66.67)$ & $1.91(0.5-7.27)$ & 0.525 \\
\hline \multicolumn{6}{|l|}{ Nerve invasion, $\mathrm{n}(\%)$} \\
\hline Yes & $21(36.84)$ & $15(33.33)$ & $6(50)$ & $0.5(0.14-1.82)$ & 0.288 \\
\hline \multicolumn{6}{|c|}{ Lymph node metastasis, n (\%) } \\
\hline Yes & $21(36.84)$ & $14(31.11)$ & $7(58.33)$ & $0.4(0.1-1.66)$ & 0.375 \\
\hline \multicolumn{6}{|l|}{ bTNM stage, n (\%) } \\
\hline$\| \mathrm{A}$ & $36(63.16)$ & $31(68.89)$ & $5(41.67)$ & $0.32(0.09-1.2)$ & 0.082 \\
\hline IIIB and IV & $21(36.84)$ & $14(31.11)$ & $7(58.33)$ & & \\
\hline
\end{tabular}

PDX patient-derived xenograft

a Chi square test

b Tumor stage was defined according to the American Joint Committee on Cancer (AJCC) TNM staging system (AJCC 7th edition)
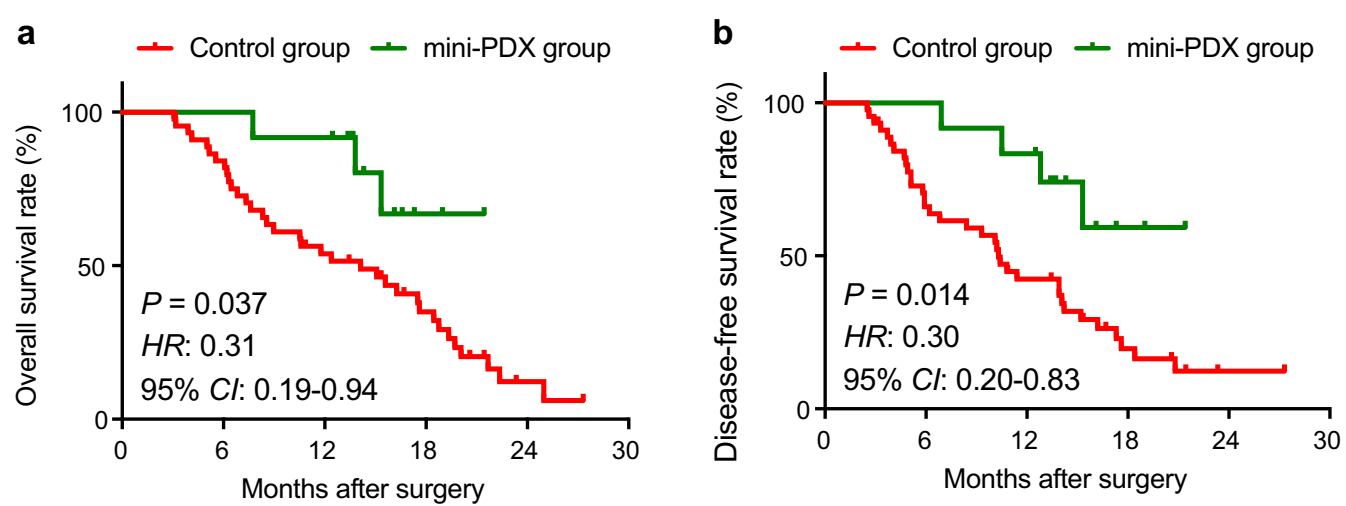

Fig. 2 Comparison of the prognosis of gallbladder carcinoma patients between the conventional chemotherapy group and the mini-PDX-guided chemotherapy group. The 12 gallbladder carcinoma patients who received agents based on the mini-PDX results had higher overall survival (a) and disease-free survival rates (b) than the 45 gallbladder carcinoma patients treated with conventional chemotherapeutic drugs. $\mathbf{b}$ The two curves were compared using log-rank test 
also had significantly longer median DFS (17.6 months; 95\% CI 14.5-20.6 months) than patients in the conventional chemotherapy group (12.0 months; $95 \% \mathrm{CI}$ 9.7-14.4 months) $(P=0.014$; HR 3.37; 95\% CI 1.67-6.79) (Fig. 2b).

\section{Correlation between drug sensitivity and clinicopathological variables and biomarkers}

Our correlation analysis revealed that gemcitabine sensitivity was correlated with nerve invasion, while irinotecan efficacy was correlated with tumor size, lymph node metastasis and TNM stage (Table 2). In our mini-PDX models, irinotecan exhibited significantly greater cytotoxic effects on gallbladder carcinoma cells with high p53 or Ki-67 expression versus those with low p53 or Ki-67 expression (Fig. 3a, b). Furthermore, nab-paclitaxel demonstrated significantly greater anti-tumor effects on gallbladder carcinoma cells with low $P$-gp expression versus those with high $P$-gp expression (Fig. 3c). Oxaliplatin demonstrated significantly greater inhibitory effects on gallbladder carcinoma cells with high MRP1, Bcl-2 or GST- $\pi$ expression; in contrast, 5-fluorouracil exhibited significantly greater inhibitory effects on gallbladder carcinoma cells with low Bcl-2 or TS expression (Fig. 3d-g).

\section{Discussion}

Cancer drug development has been hampered by a lack of preclinical models that could reliably predict the efficacy of novel compounds in cancer patients. PDX models have gained more popularity in the past several years over conventional models in predicting postoperative chemosensitivity of various tumors [19]. Here, we present the first piece of preclinical and clinical evidence for the utility of optimized mini-PDX model in guiding adjuvant chemotherapy of gallbladder cancer patients. Moreover, when prospectively comparing the efficacy of PDXguided chemotherapy versus conventional chemotherapy, we found that PDX-guided chemotherapy significantly improved the survival outcome of gallbladder carcinoma patients. Additionally, the correlations between some clinicopathological features, biomarkers and drug efficacy were also analyzed. Our present study showed that the mini-PDX models well-recapitulated the tumor behaviors of gallbladder carcinoma patients and could provide important guidance for oncologists in making informed decision on individualized chemotherapy.

Most translational cancer studies require effective preclinical models [20-23]. Human cancer models for drug screening started in the 1970s with the help of conventional cell lines. Although convenient and easy to use,

Table 2 Association analysis between clinicopathologic characteristics and chemosensitivity

\begin{tabular}{|c|c|c|c|c|c|c|c|c|c|c|}
\hline Proliferation rate (\%) & Gemcitabine & $P$ & Oxaliplatin & $P$ & 5-Fluorouracil & $P$ & Nab-paclitaxel & $P$ & Irinotecan & $P$ \\
\hline \multicolumn{11}{|l|}{ Tumor size, cm } \\
\hline$<4$ & $65.3 \pm 11.5$ & 0.316 & $72.2 \pm 27.9$ & 0.814 & $66.5 \pm 30.0$ & 0.684 & $84.8 \pm 28.5$ & 0.257 & $71.0 \pm 26.4$ & 0.049 \\
\hline$\geq 4$ & $26.8 \pm 88.6$ & & $67.3 \pm 40.2$ & & $72.8 \pm 21.7$ & & $35.0 \pm 97.4$ & & $15.0 \pm 51.9$ & \\
\hline \multicolumn{11}{|l|}{ Tumor differentiation } \\
\hline Well and moderate & $38.5 \pm 78.2$ & 0.699 & $55.0 \pm 28.7$ & 0.127 & $61.7 \pm 24.6$ & 0.292 & $35.3 \pm 67.5$ & 0.264 & $31.7 \pm 46.8$ & 0.449 \\
\hline Poor & $53.7 \pm 51.2$ & & $84.5 \pm 32.6$ & & $77.7 \pm 25.2$ & & $84.5 \pm 76.1$ & & $54.3 \pm 52.7$ & \\
\hline \multicolumn{11}{|l|}{ Nerve invasion } \\
\hline No & $10.3 \pm 72.1$ & 0.044 & $74.8 \pm 26.8$ & 0.618 & $71.2 \pm 30.2$ & 0.848 & $82.7 \pm 94.2$ & 0.303 & $37.2 \pm 56.5$ & 0.700 \\
\hline Yes & $81.8 \pm 24.8$ & & $64.7 \pm 40.4$ & & $68.2 \pm 21.9$ & & $37.2 \pm 40.9$ & & $48.8 \pm 44.8$ & \\
\hline \multicolumn{11}{|l|}{ Lymph node metastasis } \\
\hline No & $30.2 \pm 85.7$ & 0.491 & $55.4 \pm 34.3$ & 0.219 & $69.8 \pm 34.1$ & 0.989 & $21.4 \pm 66.3$ & 0.128 & $7.4 \pm 38.5$ & 0.024 \\
\hline Yes & $57.4 \pm 46.3$ & & $80.0 \pm 30.5$ & & $69.6 \pm 19.7$ & & $87.4 \pm 69.1$ & & $68.4 \pm 40.0$ & \\
\hline \multicolumn{11}{|l|}{ TNM stage } \\
\hline |-IIIA & $18.3 \pm 94.0$ & 0.305 & $61.0 \pm 36.9$ & 0.543 & $64.0 \pm 36.4$ & 0.606 & $13.3 \pm 73.6$ & 0.122 & $-7.5 \pm 22.2$ & 0.004 \\
\hline IIIB-IV & $60.0 \pm 43.5$ & & $74.1 \pm 32.7$ & & $72.5 \pm 20.0$ & & $83.3 \pm 65.1$ & & $68.3 \pm 37.0$ & \\
\hline
\end{tabular}

$P$ values were calculated by unpaired $t$-test (2-sided) 
a

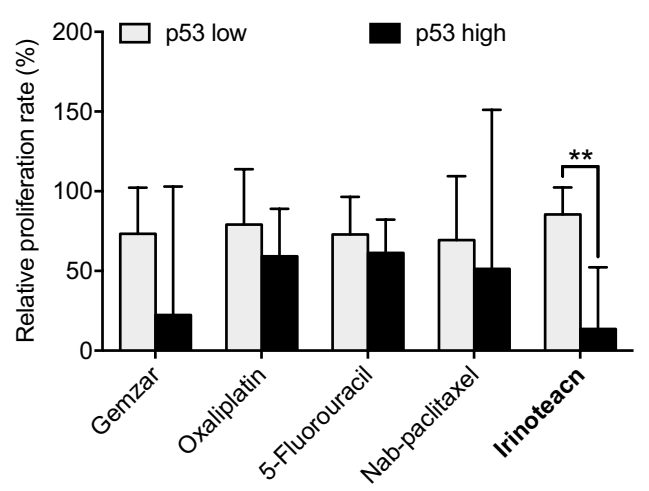

C

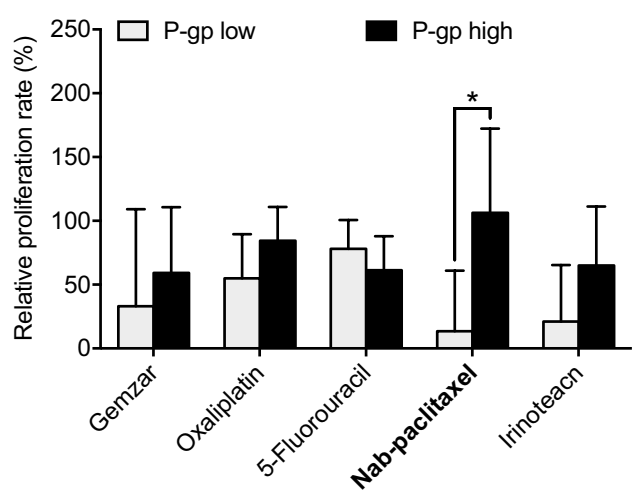

e

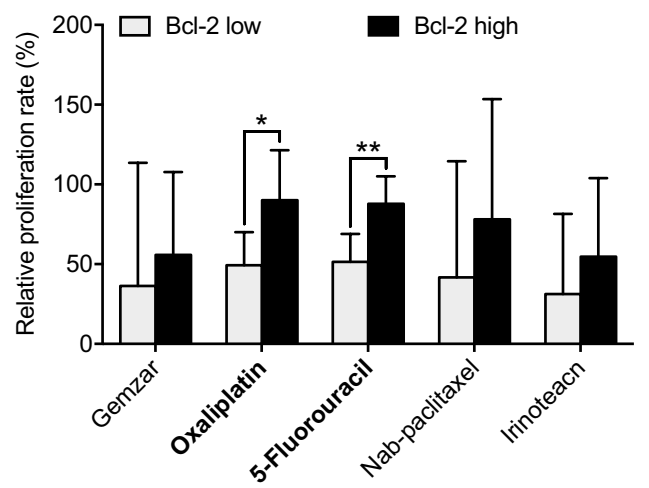

g

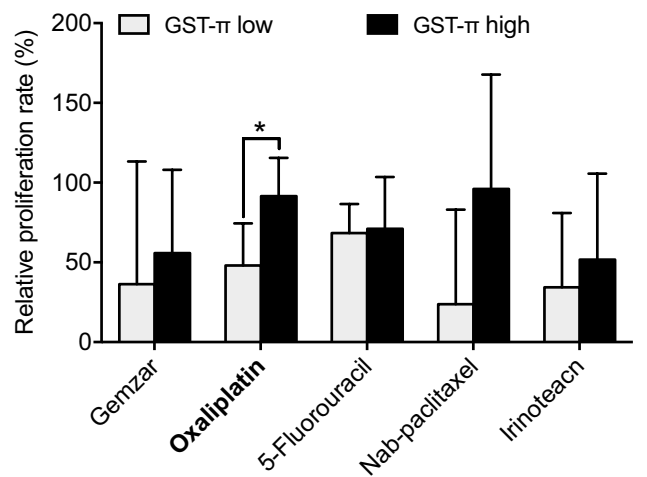

b

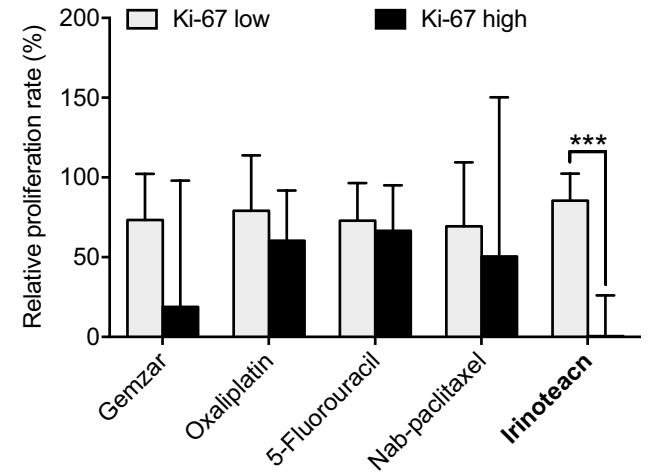

d

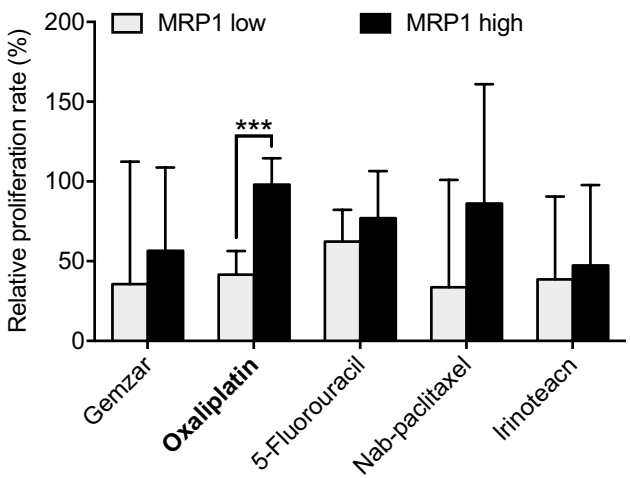

f

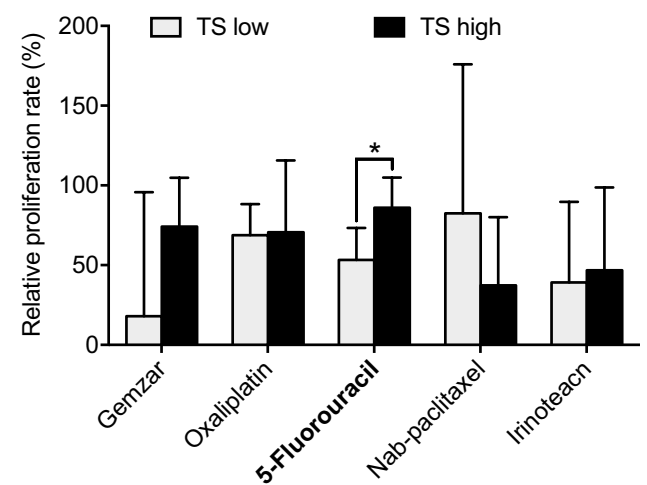

h

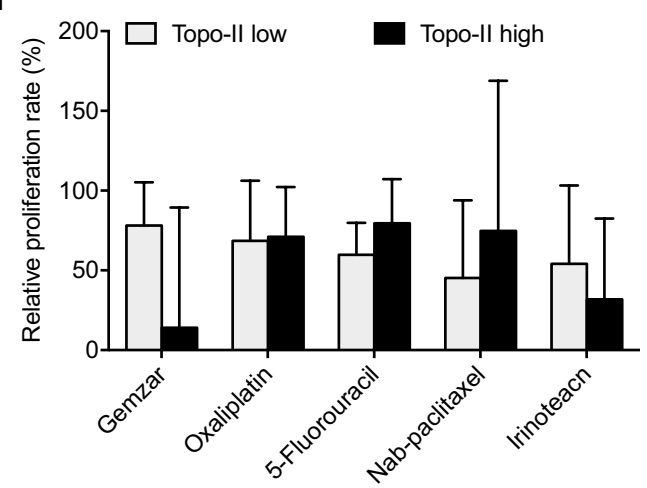


these cell lines-based studies lack the predictive value of specific cancer types for clinical application. The preclinical PDX models has circumvent the limitations of conventional cell line-based models and are now more commonly used. These models can provide drug sensitivities that mimic the clinical response of cancer patients to cytotoxic agents [24]. Also, since PDX models correlate well with the pathologic characteristics and genetic features of the tumors of individual patients, they are becoming the preferred preclinical tool to improve the drug development process [24]. However, the long time in establishing PDX models restrains their usage in more aggressive cancers like gallbladder carcinoma; not to mention that several transplantation cycles are needed for tumor xenograft formation in PDX models and which might alter the properties of the originally transplanted tumor. Reports have demonstrated that the finally formed tumor xenograft is subsequently a more aggressive phenotype and behaves more like metastatic tumor [25]. Most gallbladder carcinoma patients are diagnosed at advanced stages, and thus require prompt initiation of anti-tumor therapy. In this perspective, mini-PDX model is the more suitable alternative.

Currently, gemcitabine, oxaliplatin, 5-fluorouracil, nab-paclitaxel, and irinotecan are the five most potentially effective agents for gallbladder carcinoma patients [26]. Single or combination chemotherapies of these drugs have shown improved median survival rates of gallbladder carcinoma patients. However, further clinical application of these drugs are often impeded by the uncommon nature of gallbladder carcinoma and efficacy data are mostly based on studies from other biliary tract tumors like intrahepatic or extrahepatic cholangiocarcinoma, which are now considered to be different from gallbladder carcinoma [12, 27]. In this study, an improved prognosis was observed when the effectiveness of five chemotherapeutic drugs were separately tested using mini-PDX models, after which the two most effective drugs identified were prospectively prescribed to gallbladder carcinoma patients. This personalized treatment provides a scientific rationale for clinical therapy and avoids the side effects from clinical experience-guided medication. Aside from cytotoxic chemotherapeutic agents, several targeted agents against EGFR, VEGF or MEK have also been reported to be useful in treating gallbladder cancer. Therefore, our mini-PDX model could be suitable for preclinical testing of the effectiveness of these drugs in the coming future.

Aside from providing reliable references for the choice of drugs clinically, the mini-PDX models, when combined with other technical methods, could also accelerate the understanding of the underlying mechanisms of oncogenesis, tumor progression and chemoresistance. In this present study, we have found that gemcitabine sensitivity was correlated with nerve invasion, and irinotecan efficacy was associated with tumor size, lymph node metastasis and TNM stage. Future studies with a larger sample set are required to confirm these relationships and help elucidate the underlying mechanisms. P53, Ki-67, P-gp, MRP1, Bcl2 , TS, GST- $\pi$ and Topo-II are important in modulating chemosensitivity $[28,29]$. The reactivity to irinotecan in our mini-PDX model was found to correlate with p53 and Ki-67 expression in gallbladder carcinoma patients. Oxaliplatin sensitivity was associated with MRP1 and $\mathrm{Bcl}-2$ expression. These findings demonstrate that the mini-PDX model is effective in predicting chemosensitivity of gallbladder carcinoma patients and lends support to the future application of this method in clinical practice. In addition, based on our obtained results, chemo resistant and chemosensitive PDX tumor models could be developed to study the molecular mechanisms of chemoresistance in gallbladder carcinoma, which would be conducive in finding potential therapeutic targets and predictive markers of chemosensitivity for gallbladder cancer patients. Hence, mini-PDX models has potential prospect of being extended in the treatment of other types of malignant tumors.

\section{Conclusions}

Our results show that the mini-PDX model is an effective tool in guiding the choice of chemotherapeutic regimens for gallbladder carcinoma patients and that PDX-guided chemotherapy can significantly improve the survival of gallbladder carcinoma patients compared to conventional chemotherapy. However, further confirmations from studies with larger sample size are needed.

\section{Abbreviations \\ PDX: patient-derived xenograft; OS: overall survival; DFS: disease free survival rate; HBSS: Hank's balanced salt solution; RFU: relative fluorescence units; SD: standard deviation.}

\section{Authors' contributions}

JW was responsible for coordination of the project and contributed to the study design. The writing team consisted of JW, MZ, and RMY. MZ, RMY, MH, and WC performed most of in vitro and in vivo experiments. $M H, L H Y$, and $H W$ took part in the collection of clinical samples and features. QL and MML performed IHC data analysis. All authors read and approved the final manuscript.

\footnotetext{
Author details

${ }^{1}$ Department of Biliary-Pancreatic Surgery, Renji Hospital, School of Medicine, Shanghai Jiao Tong University, 160 Pujian Road, Shanghai 200127, P. R. China.

${ }^{2}$ Department of Pathology, Renji Hospital, School of Medicine, Shanghai Jiao Tong University, Shanghai 200127, P. R. China. ${ }^{3}$ Department of Pathology, Shanghai Ninth People's Hospital, School of Medicine, Shanghai Jiao Tong University, Shanghai 200011, P. R. China.
} 


\section{Acknowledgements}

We appreciate Dr. Wen Danyi (LIDE Biotech Inc.) for providing technical support on mini-PDX models.

\section{Competing interests}

The authors declare that they have no competing interests.

\section{Availability of data and materials}

The datasets used and analyzed during the current study are available from the corresponding author on reasonable request.

\section{Consent for publication}

All subjects provided written informed consent before enrollment and agreed to publication of their individual data.

\section{Ethics approval and consent to participate}

This study was approved by Ethical Committee of Renji Hospital, School of Medicine, Shanghai Jiao Tong University.

\section{Funding}

This work was supported by the Foundation of Shanghai Shen Kang Hospital Development Center (Nos. 16CR2002A and 16CR3028A), National Science Foundation of China (Nos. 81472240 and 81773184), and Shanghai Outstanding Academic Leaders Plan (2016, JW).

\section{Received: 21 December 2017 Accepted: 6 July 2018}

Published online: 17 July 2018

\section{References}

1. de Groen PC, Gores GJ, LaRusso NF, Gunderson LL, Nagorney DM. Biliary tract cancers. N Engl J Med. 1999;341(18):1368-78. https://doi. org/10.1056/NEJM199910283411807.

2. Lazcano-Ponce EC, Miquel JF, Munoz N, Herrero R, Ferrecio C, Wistuba II, et al. Epidemiology and molecular pathology of gallbladder cancer. CA Cancer J Clin. 2001;51(6):349-64.

3. Miller KD, Siegel RL, Lin CC, Mariotto AB, Kramer JL, Rowland JH, et al. Cancer treatment and survivorship statistics, 2016. CA Cancer J Clin. 2016:66(4):271-89. https://doi.org/10.3322/caac.21349.

4. Hueman MT, Vollmer CM Jr, PawlikTM. Evolving treatment strategies for gallbladder cancer. Ann Surg Oncol. 2009;16(8):2101-15. https://doi. org/10.1245/s10434-009-0538-x.

5. Chan E, Berlin J. Biliary tract cancers: understudied and poorly understood. J Clin Oncol. 2015;33(16):1845-8. https://doi.org/10.1200/ JCO.2014.59.7591.

6. Huang Y, Li X, Zhao Y. Progression of targeted therapy in advanced cholangiocarcinoma. Chin J Cancer Res. 2015;27(2):122-7. https://doi. org/10.3978/j.issn.1000-9604.2015.04.01.

7. Lee J, Park SH, Chang HM, Kim JS, Choi HJ, Lee MA, et al. Gemcitabine and oxaliplatin with or without erlotinib in advanced biliary-tract cancer: a multicentre, open-label, randomised, phase 3 study. Lancet Oncol. 2012:13(2):181-8. https://doi.org/10.1016/S1470-2045(11)70301-1.

8. Jensen LH. Biliary-tract cancer: improving therapy by adding molecularly targeted agents. Lancet Oncol. 2012;13(2):118-9. https://doi.org/10.1016/ S1470-2045(11)70329-1.

9. Endlicher E, Schnoy E, Troppmann M, Rogler G, Messmann H, Klebl F, et al. Irinotecan plus gemcitabine and fluorouracil in advanced biliary tract cancer: a retrospective study. Digestion. 2016:93(3):229-33. https://doi. org/10.1159/000445187

10. Chung MJ, Kim YJ, Park JY, Bang S, Song SY, Chung JB, et al. Prospective phase II trial of gemcitabine in combination with irinotecan as first-line chemotherapy in patients with advanced biliary tract cancer. Chemotherapy. 2011;57(3):236-43. https://doi.org/10.1159/000328021.

11. Williams TM, Majithia L, Wang SJ, Thomas CR Jr. Defining the role of adjuvant therapy: cholangiocarcinoma and gall bladder cancer. Semin Radiat Oncol. 2014;24(2):94-104. https://doi.org/10.1016/j.semra donc.2014.01.001.
12. Nakamura H, Arai Y, Totoki Y, Shirota T, Elzawahry A, Kato M, et al. Genomic spectra of biliary tract cancer. Nat Genet. 2015;47(9):1003-10. https://doi. org/10.1038/ng.3375.

13. Valle JW, Lamarca A, Goyal L, Barriuso J, Zhu AX. New horizons for precision medicine in biliary tract cancers. Cancer Discov. 2017;7(9):943-62. https://doi.org/10.1158/2159-8290.CD-17-0245.

14. Gandara DR, Lara PN Jr, Mack PC. Patient-derived xenografts for investigation of acquired resistance in oncogene-driven cancers: building a better mousetrap. J Clin Oncol. 2015;33(26):2839-40. https://doi.org/10.1200/ JCO.2015.61.9692

15. Bissig-Choisat B, Kettlun-Leyton C, Legras XD, Zorman B, Barzi M, Chen LL, et al. Novel patient-derived xenograft and cell line models for therapeutic testing of pediatric liver cancer. J Hepatol. 2016;65(2):325-33. https://doi. org/10.1016/j.jhep.2016.04.009.

16. Chapuy B, Cheng H, Watahiki A, Ducar MD, Tan Y, Chen L, et al. Diffuse large B-cell lymphoma patient-derived xenograft models capture the molecular and biological heterogeneity of the disease. Blood. 2016;127(18):2203-13. https://doi.org/10.1182/blood-2015-09-672352.

17. Nicolle D, Fabre M, Simon-Coma M, Gorse A, Kappler R, Nonell L, et al. Patient-derived mouse xenografts from pediatric liver cancer predict tumor recurrence and advise clinical management. Hepatology. 2016;64(4):1121-35. https://doi.org/10.1002/hep.28621.

18. Yang RM, Zhan M, Xu SW, Long MM, Yang LH, Chen W, et al. miR-3656 expression enhances the chemosensitivity of pancreatic cancer to gemcitabine through modulation of the RHOF/EMT axis. Cell Death Dis. 2017;8(10):e3129. https://doi.org/10.1038/cddis.2017.530.

19. Tentler JJ, Tan AC, Weekes CD, Jimeno A, Leong S, Pitts TM, et al. Patientderived tumour xenografts as models for oncology drug development. Nat Rev Clin Oncol. 2012;9(6):338-50. https://doi.org/10.1038/nrcli nonc.2012.61

20. Hu X, Chen L, Du Y, Fan B, Bu Z, Wang X, et al. Postoperative chemotherapy with S-1 plus oxaliplatin versus S-1 alone in locally advanced gastric cancer (RESCUE-GC study): a protocol for a phase III randomized controlled trial. Chin J Cancer Res. 2017;29(2):144-8. https://doi.org/10.21147 /j.issn.1000-9604.2017.02.07.

21. Hu X, Wang L, Lin L, Han X, Dou G, Meng Z, et al. A phase I trial of an oral subtype-selective histone deacetylase inhibitor, chidamide, in combination with paclitaxel and carboplatin in patients with advanced non-small cell lung cancer. Chin J Cancer Res. 2016;28(4):444-51. https://doi. org/10.21147/j.issn.1000-9604.2016.04.08.

22. Venditti JM, Wesley RA, Plowman J. Current NCI preclinical antitumor screening in vivo: results of tumor panel screening, 1976-1982, and future directions. Adv Pharmacol Chemother. 1984;20:1-20.

23. Zhou $H$, Song $Y$, Jiang J, Niu H, Zhao H, Liang J, et al. A pilot phase II study of neoadjuvant triplet chemotherapy regimen in patients with locally advanced resectable colon cancer. Chin J Cancer Res. 2016;28(6):598_605. https://doi.org/10.21147/j.issn.1000-9604.2016.06.06.

24. Choi SY, Lin D, Gout PW, Collins CC, Xu Y, Wang Y. Lessons from patientderived xenografts for better in vitro modeling of human cancer. Adv Drug Deliv Rev. 2014;79-80:222-37. https://doi.org/10.1016/j. addr.2014.09.009.

25. Lai Y, Wei X, Lin S, Qin L, Cheng L, Li P. Current status and perspectives of patient-derived xenograft models in cancer research. J Hematol Oncol. 2017;10(1):106. https://doi.org/10.1186/s13045-017-0470-7.

26. Horgan AM, Amir E, Walter T, Knox JJ. Adjuvant therapy in the treatment of biliary tract cancer: a systematic review and meta-analysis. J Clin Oncol. 2012;30(16):1934-40. https://doi.org/10.1200/JCO.2011.40.5381.

27. Razumilava N, Gores GJ. Building a staircase to precision medicine for biliary tract cancer. Nat Genet. 2015;47(9):967-8. https://doi.org/10.1038/ ng.3386.

28. Xue $X$, Liang XJ. Overcoming drug efflux-based multidrug resistance in cancer with nanotechnology. Chin J Cancer. 2012;31(2):100-9. https://doi. org/10.5732/cjc.011.10326.

29. Zhang JT. Use of arrays to investigate the contribution of ATP-binding cassette transporters to drug resistance in cancer chemotherapy and prediction of chemosensitivity. Cell Res. 2007;17(4):311-23. https://doi. org/10.1038/cr.2007.15 